\title{
Análise morfológica dos fissurados de lábio e palato do Centro de Tratamento de Anomalias Craniofaciais do Estado do Rio de Janeiro
}

Alexandre de Almeida Ribeiro*, Luíse Leal**, Rawlson de Thuin***

\begin{abstract}
Resumo
Objetivo: caracterizar o padrão oclusal e facial para diagnosticar as necessidades reabilitadoras dos fissurados de lábio e palato do Centro de Tratamento de Anomalias Craniofaciais do Estado do Rio de Janeiro. Metodologia: vinte indivíduos, com fissura transforame incisivo unilateral, operados de lábio e palato, sem histórico de tratamento ortodôntico, foram avaliados, por meio de modelos de gesso superior, quanto à dimensão transversa e forma do arco superior, assim como a presença de mordidas cruzadas, com os modelos em MIH. O padrão facial, analisado por meio de fotografias de perfil, e a presença de enxerto ósseo na área da fissura, diagnosticada através de radiografias oclusais, foram também determinados. Os dados referentes à dimensão transversa foram confrontados com as médias de um grupo com oclusão normal ( $\mathrm{n}=22)$. Resultados: a comparação indicou forte atresia do arco superior associada a diferentes mordidas cruzadas na amostra fissurada. Já o padrão facial revelou clara deficiência do terço médio, enquanto o enxerto ósseo alveolar não se fez presente no processo de reabilitação. Conclusão: a atresia maxilar em associação com as mordidas cruzadas são fatos que comprovam o impacto negativo das cirurgias de lábio e palato no crescimento tridimensional da maxila. A busca pelo padrão ouro de reabilitação não será possível sem o enxerto ósseo, uma modalidade cirúrgica que confere unidade à base óssea maxilar e provê integridade ao rebordo alveolar.
\end{abstract}

Palavras-chave: Fissura labiopalatal. Morfologia maxilar. Dimensão transversa maxilar.

\section{INTRODUÇÃO}

O consenso de interdisciplinaridade vigente no tratamento dos pacientes fissurados de lábio e palato se consolidou na segunda metade do século XX e, desde então, enfatiza a complexidade do processo reabilitador, que se inicia no nascimento e se estende até a idade adulta, com o término do crescimento craniofacial ${ }^{1,14,15}$. O objetivo final é a busca pela reabilitação estética e funcional, que visa, fundamentalmente, a reintegração social dos portadores de fissuras de lábio e palato, estigmatizados ao nascimento pela anomalia estampada na face ${ }^{2}$.

* Ortodontista do Centro de Tratamento de Anomalias Craniofaciais do Rio de Janeiro - REVIVA. Mestre e Especialista em Ortodontia pela Universidade Federal do Rio de Janeiro (UFRJ).

** Coordenadora do Núcleo de Ensino e Pesquisa (NEP) do REVIVA. Mestre em Materiais Dentários pela Universidade de São Paulo (USP). Doutora em Materiais Preventivos e Restauradores pela Universidade Federal do Rio de Janeiro (UFRJ).

*** Diretor Geral do Centro de Tratamento de Anomalias Craniofaciais do Rio de Janeiro - REVIVA. Cirurgião Plástico Titular da Sociedade Brasileira de Cirurgia Plástica (SBCP). 
A filosofia da interdisciplinaridade na reabilitação envolve, primordialmente, um corpo clínico experiente com a mentalidade voltada para um protocolo de tratamentos seqüenciais específicos, que, necessariamente, estabeleça as prioridades do paciente de acordo com a fase de desenvolvimento na qual ele se encontra ${ }^{2}, 12,17,18,19,20,21,22,23$.

Segundo o protocolo do Hospital de Reabilitação de Anomalias Craniofaciais da Universidade de São Paulo (HRAC/USP), referência no Brasil e América Latina para o tratamento de fissurados, as cirurgias reparadoras primárias de reconstrução do lábio e palato devem preferencialmente se iniciar no primeiro ano de vida $2,7,8,9,12,13,17,18,19,20,22,23,28$. Pelo protocolo ideal, o lábio deve ser operado a partir dos 3 meses de idade, em procedimento conhecido como queiloplastia, enquanto 12 meses é o tempo determinado para a palatoplastia ${ }^{8,12,17,22}$.

A definição das etapas operatórias foi moldada de acordo com o protocolo de intenções do The Center for Craniofacial Anomalies at the University of Illinois ${ }^{1,2,14,15}$. A filosofia de tratamento foi estabelecida a partir das diretrizes preconizadas por dois pesquisadores da instituição, os pioneiros nos estudos em longo prazo do crescimento craniofacial em fissurados: os ortodontistas e professores Dr. Pruzansky e Dr. Addus ${ }^{1,14}$.

Cirurgias secundárias a partir dos quatro anos de vida são geralmente necessárias para retoques labiais e da asa do nariz, normalmente deslocada pela ausência de suporte ósseo nas fissuras que envolvem o rebordo alveolar ${ }^{11,27}$. A palatoplastia secundária, também nesse período, busca a otimização da função velo-faríngea, pelo restabelecimento da função muscular do palato mole, não alcançada integralmente no ato cirúrgico primário ${ }^{23}$.

Toda essa manipulação cirúrgica gera restrição tridimensional do crescimento maxilar. Esse fator ambiental potencializa a deficiência maxilar inerente à segmentação da maxila ${ }^{18,22,23,29}$. Independente da habilidade do cirurgião plástico e das técnicas atualmente utilizadas, a alteração no desenvolvimento maxilofacial repercute no perfil do fissurado, que tende à concavidade, pela deficiência sagital do terço médio ${ }^{1,2,7,8,9,12,14,15,17,18,20,22,23,29}$. No plano transverso, a atresia maxilar se associa à mordida cruzada de diferentes magnitudes pela

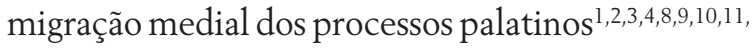
13,18,22,23,29 (colapso maxilar), enquanto nota-se um aumento na altura facial pelo padrão de crescimento mandibular ${ }^{28}$.

Em longo prazo o deslocamento medial dos segmentos palatinos pode criar três situações morfológicas distintas ${ }^{1,12,14,15}$ : 1) aproximação e contato dos segmentos palatinos, originando arcos aparentemente simétricos; 2) colapso dos segmentos palatinos, com o segmento menor dentro do maior, e 3) aproximação sem contato dos segmentos palatinos.

Essas alterações acompanham a fase de crescimento maxilar e progridem em complexidade conforme a idade do paciente ${ }^{10,11}$. O crescimento da maxila se acelera no início da dentadura mista, por volta de 6 a 7 anos, que justamente por isso é a fase ideal para o início da intervenção ortodôntica ${ }^{2,7,8,9,10,}$ 17,18,19,20,22,23. Após esse período, as manifestações do crescimento alterado passarão a agredir impiedosamente a maxila, iniciando a estigmatização facial dos fissurados. Cabe, única e exclusivamente, ao ortodontista a triste tarefa de dirimir os efeitos maléficos do crescimento facial alterado, caracterizado no aspecto intrabucal pelas mordidas cruzadas posterior e anterior, características freqüentes nos fissurados de lábio e palato $3,7,9$.

A primeira etapa do tratamento ortodôntico (Ortodontia pré-enxerto ósseo alveolar) é de caráter expansionista essencial para a correção da mordida cruzada posterior e fundamental para o restabelecimento das dimensões transversas da maxila atrésica. O disjuntor maxilar preconizado por Haas e modificado por Silva Filho é o principal dispositivo utilizado para o reposicionamento lateral dos processos maxilares colapsados $7,17,19$. Nesta fase, a tração reversa da maxila pode ser indicada, simultaneamente ao período de contenção pós-expansão, para reverter casos de discrepância sagital negativa, 
constatada pela mordida cruzada anterior ${ }^{17}$.

Além da reorganização das dimensões transversas, o restabelecimento ortopédico do contorno anatômico maxilar favorece a realização da cirurgia de enxerto ósseo alveolar autógeno da crista ilíaca $2,5,7,16,20,24,25$. Conduta adotada a partir dos promissores resultados obtidos pela Universidade de Oslo, o enxerto ósseo estabelece a união entre os processos palatinos fendidos e confere unidade à base óssea maxilar ${ }^{5,24,25}$. O tempo exato para a sua realização é determinado pelo desenvolvimento intra-ósseo do canino permanente adjacente à fissura. Quando este se encontra com 2/3 de sua raiz formada, normalmente entre os 8 e 12 anos de idade, o enxerto deve ser executado para prover estrutura óssea alveolar ao canino ao final de seu movimento eruptivo ${ }^{5,24,25}$. Por ser realizado ao final do segundo período transitório da dentadura mista, este procedimento recebeu a denominação de enxerto ósseo alveolar secundário, com vantagens prognósticas relatadas por diversos trabalhos científicos $2,5,7,16,20,24,25$.

Após 90 dias, tempo mínimo para a consolidação e integração óssea do enxerto ${ }^{5,7,16,20}$, tem início a Ortodontia pós-enxerto, caracterizada pelo alinhamento e nivelamento dentário. $\mathrm{O}$ objetivo é prover inclinação adequada às raízes adjacentes à fissura, reabilitando a oclusão, sem a necessidade de intervenção protética na área do incisivo lateral, que geralmente se encontra ausente $e^{2,7,12,17}$.

Esse protocolo de intenções busca estabelecer o padrão ouro na reabilitação funcional, estética e social dos fissurados, a qual deve se iniciar precocemente e ser acompanhada até o final do crescimento. No entanto, nem sempre é possível estender o ideal à grande população de fissurados, principalmente em regiões carentes de atendimento especializado para tal fim, entre elas o estado do Rio de Janeiro.

O Centro de Tratamento de Anomalias Craniofaciais do Rio de Janeiro - REVIVA foi criado em 2003 com o objetivo de suprir a demanda reprimida no estado fluminense, no tratamento reabilitador para o fissurado lábio-palatal. Essa re- pressão no tratamento cria peculiaridades à população portadora dessa anomalia, entre elas a alta idade dos pacientes, em sua maioria adultos, com necessidades de reabilitação que diferem do paciente infanto-juvenil.

Para tanto, o objetivo desse trabalho foi avaliar as características oclusais e faciais de fissurados de lábio e palato em tratamento no REVIVA, no intuito de determinar as necessidades intra e extrabucais para uma oclusão satisfatória, dentro do ideal preconizado pelo Hospital de Reabilitação de Anomalias Craniofaciais da Universidade de São Paulo (HRAC / USP).

\section{MATERIAL E MÉTODOS Caracterização da amostra de fissurados em relação ao grupo normal}

Foram selecionados pacientes do setor de Ortodontia do REVIVA que se enquadravam, segundo a classificação de Spina ${ }^{21,26}$, em fissurados do tipo TransForame Incisivo Unilateral - TFIU (Tab. 1). A amostra totalizou 20 indivíduos do gênero masculino, com média de idade de 24 anos e 2 meses (Tab. 2), anteriormente já operados de lábio e palato. Os modelos superiores de gesso de cada um dos pacientes foram utilizados para análise da dimensão maxilar transversa, realizada com paquímetro em quatro regiões do arco superior: 1) entre caninos (3-3), 2) entre primeiros pré-molares (4-4), 3) entre segundos pré-molares (5-5) e 4) entre primeiros molares (6-6).

As pontas de cúspides dos caninos, pontas de cúspides vestibulares de primeiros e segundos prémolares e pontas de cúspides mesiovestibulares de primeiros molares serviram como parâmetro para medição entre os dentes contralaterais (Fig. 1).

Os dados obtidos foram confrontados com as respectivas dimensões médias do grupo controle, composto de 22 pacientes normais do gênero masculino, com idade média de 19 anos e 5 meses, provenientes do Hospital de Reabilitação de Anomalias Craniofaciais da Universidade de São Paulo - HRAC/USP, publicadas em artigo anterior ${ }^{23}$. 
Tabela 1 - Distribuição do grupo amostral de pacientes com fissura Transforame Incisivo Unilateral (TFIU), segundo a localização da fissura: direita (TFIUD) e esquerda (TFIUE).

\begin{tabular}{cc}
\hline \multicolumn{1}{c}{ grupo amostral - TFIU } \\
\hline TFIUD & 9 \\
TFIUE & 11 \\
$\mathrm{n}$ (total) & 20 \\
\hline
\end{tabular}
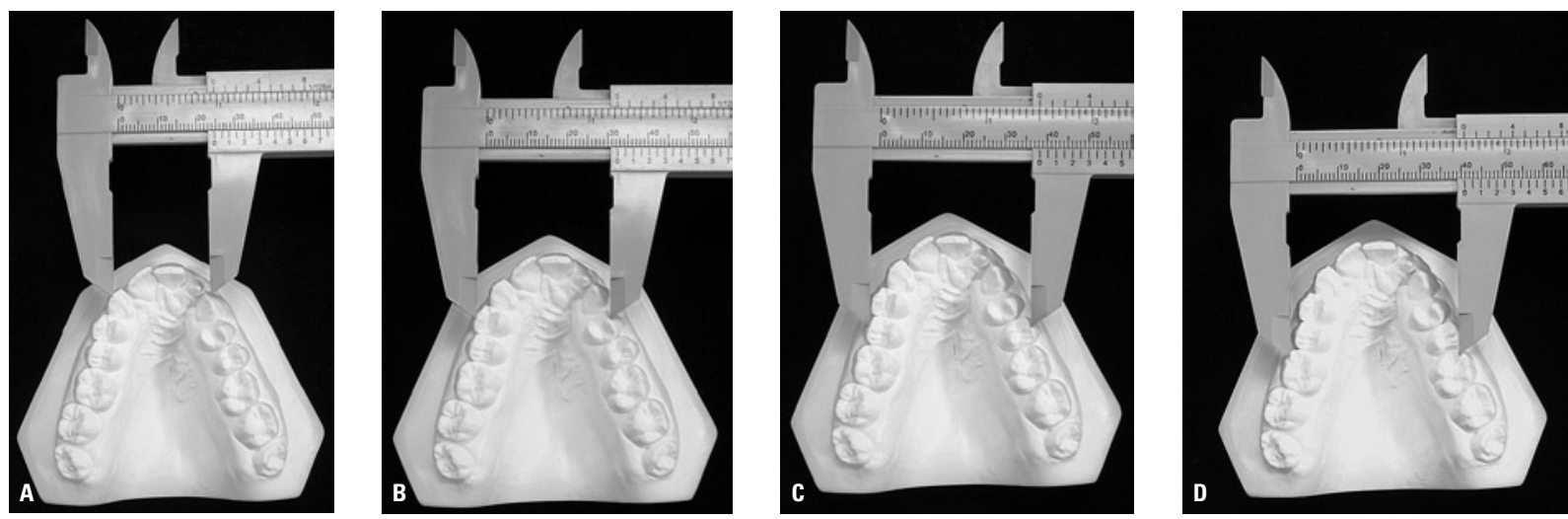

FIGURA 1 - Obtenção da dimensão transversa com auxílio de paquímetro: A) entre caninos, B) entre primeiros pré-molares, C) entre segundos pré-molares D) entre primeiros molares.

\section{Perfil oclusal do grupo fissurado}

Por meio dos modelos de gesso do arco dentário superior, foi determinado morfologicamente o tipo de arco maxilar, segundo Aduss ${ }^{1}$ e Pruzansky $^{14}$, de acordo com o padrão de medialização dos processos palatinos (Fig. 2), classificados da seguinte maneira: 1) migração com contato dos segmentos palatinos, 2) migração sem contato dos segmentos palatinos, 3) segmentos colapsados.

A relação de má oclusão do arco dentário superior em relação ao inferior foi verificada, no sentido transverso e sagital, pela presença de mordida cruzada (Fig. 3), determinada pela análise dos modelos em máxima intercuspidação habitual (MIH) e agrupados, a seguir, em: 1) anterior, 2) posterior unilateral, 3) posterior bilateral, 4) ântero-posterior unilateral e 5) total.

Por meio de fotografias de perfil, a amostra foi, ainda, classificada morfologicamente segundo o padrão facial, como preconizado por $\mathrm{Ca}$ pelozza Filho ${ }^{6}$ (Fig. 4), e agrupada da seguinte
Tabela 2 - Idade de início da terapia ortodôntica.

\begin{tabular}{|c|c|c|c|}
\hline \multicolumn{4}{|c|}{ idade início Ortodontia ( $n=20)$} \\
\hline média & mínima & máxima & d.p. \\
\hline $24 a \mathrm{am}$ & 10a $2 \mathrm{~m}$ & $46 \mathrm{a} 1 \mathrm{~m}$ & 8,45 \\
\hline
\end{tabular}

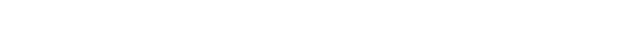




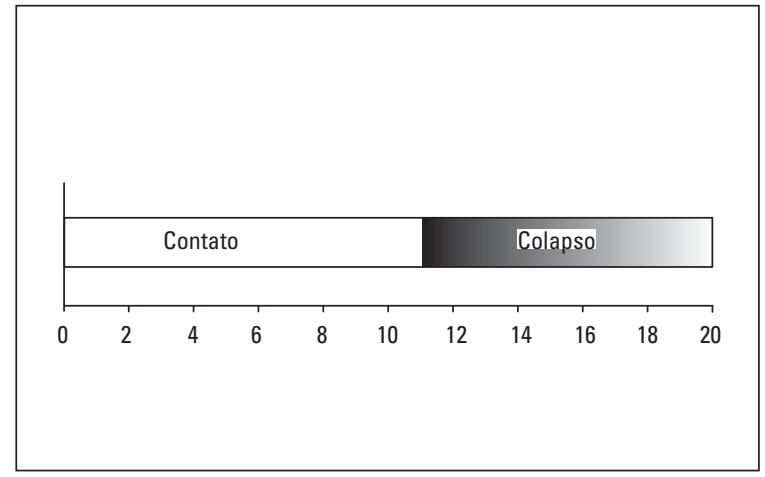

GRÁFICO 1 - Tipos de migração medial dos processos palatinos segundo Aduss $^{1}$ e Pruzansky ${ }^{14}$ observadas em cada um dos pacientes. Não foi observada a migração sem contato dos segmentos fissurados.

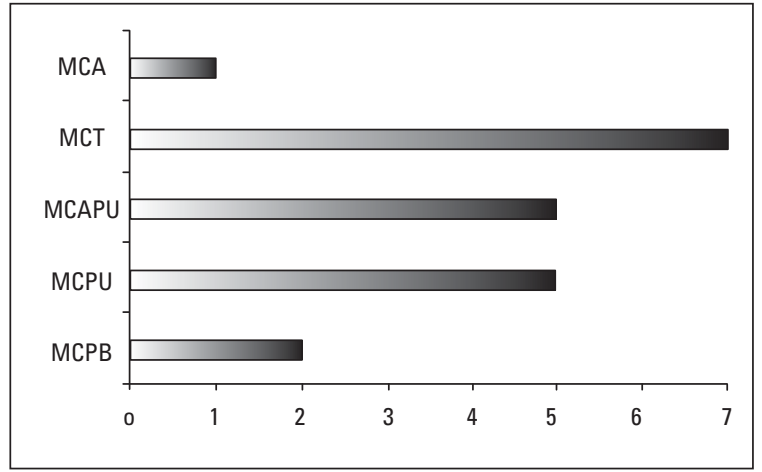

GRÁFICO 2 - Presença de mordida cruzada, determinada pela análise dos modelos em máxima intercuspidação habitual (MIH) em números absolutos: Mordida Cruzada Anterior (MCA), Mordida Cruzada Total (MCT), Mordida Cruzada Ântero-Posterior Unilateral (MCAPU), Mordida Cruzada Posterior Unilateral (MCPU) e Mordida Cruzada Posterior Bilateral (MCPB).
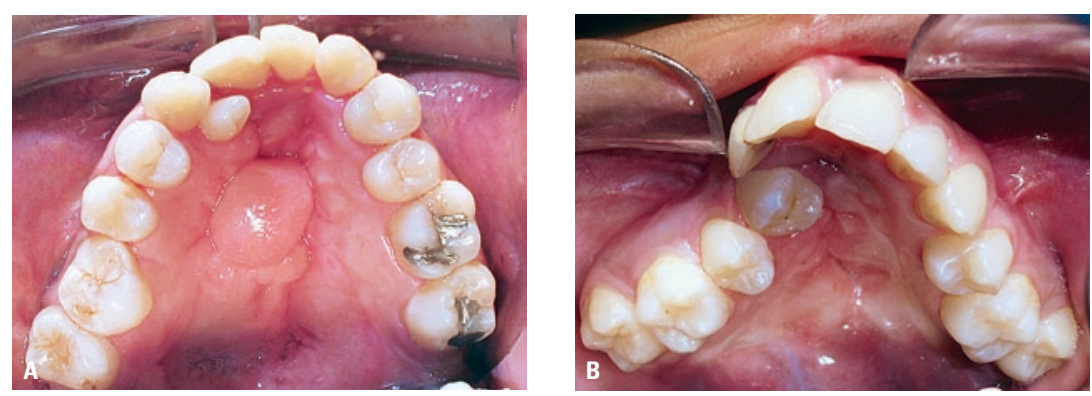

FIGURA 2 - Tipos de arco encontrados, classificados segundo Aduss ${ }^{1}$ e Pruzansky ${ }^{14}$. A) Migração com contato dos segmentos palatinos, criando arco aparentemente simétrico, B) arco colapsado, com o segmento menor dentro do maior.
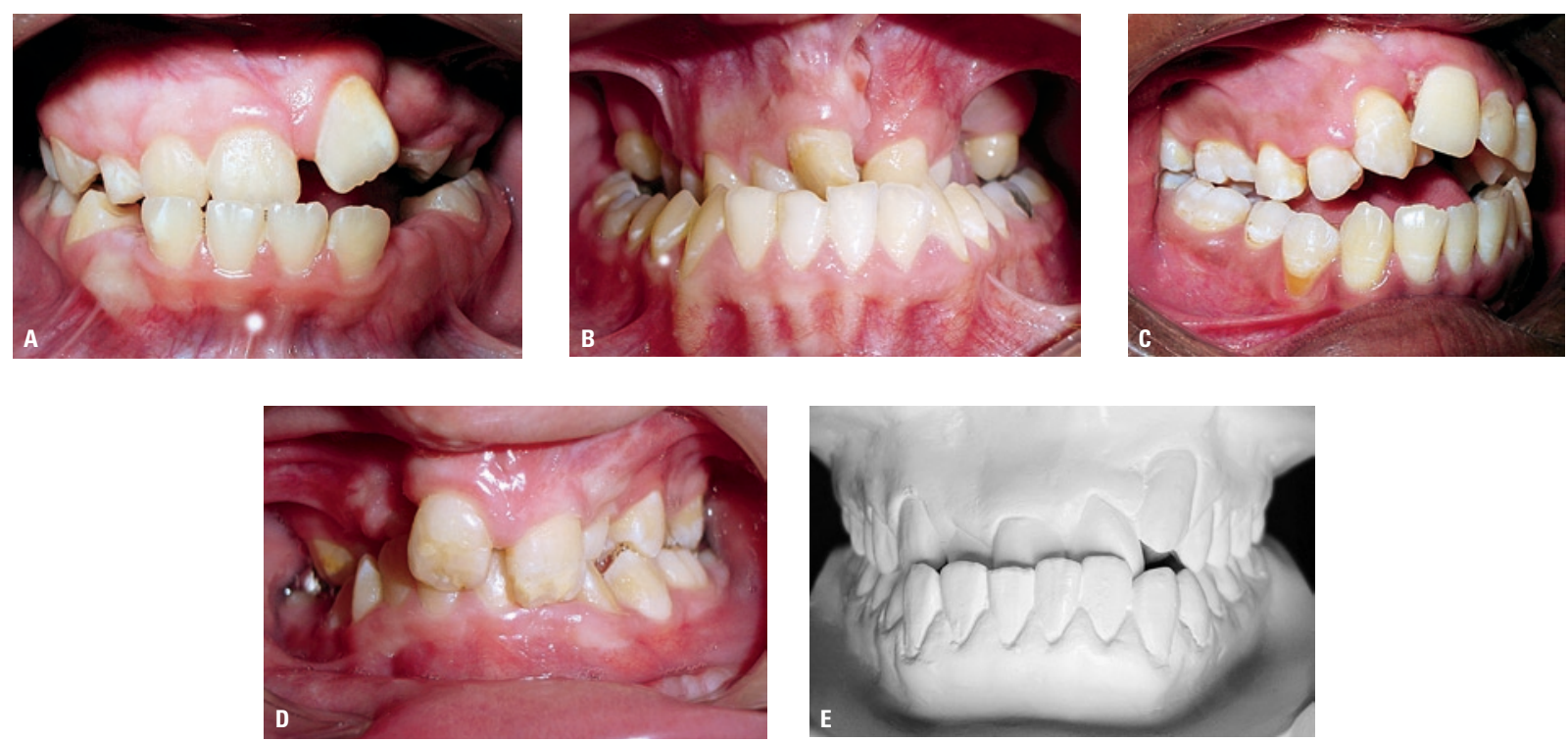

FIGURA 3 - Mordida Cruzada A) ântero-posterior unilateral, B) total, C) posterior unilateral, D) posterior bilateral, E) anterior. 


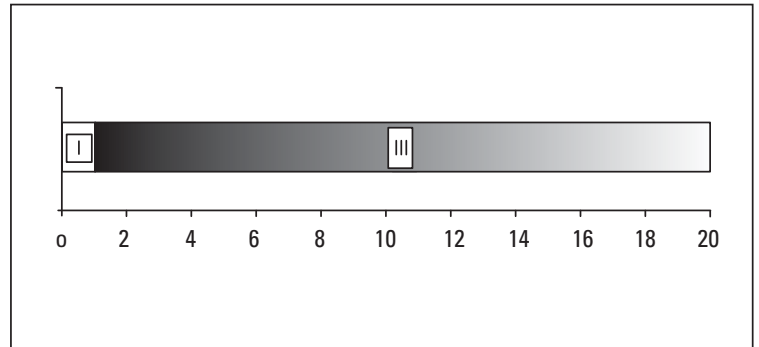

GRÁFICO 3 - Classificação do padrão facial de acordo com a relação sagital entre as bases ósseas superior e inferior. Como esperado, não se observou o padrão facial II.

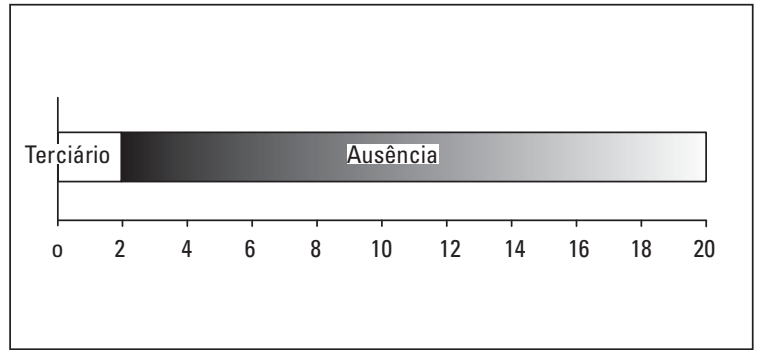

GRÁFICO 4 - Ocorrência do enxerto ósseo na área da fissura, classificado de acordo com o tempo cirúrgico. Não foi constatado o enxerto ósseo secundário.
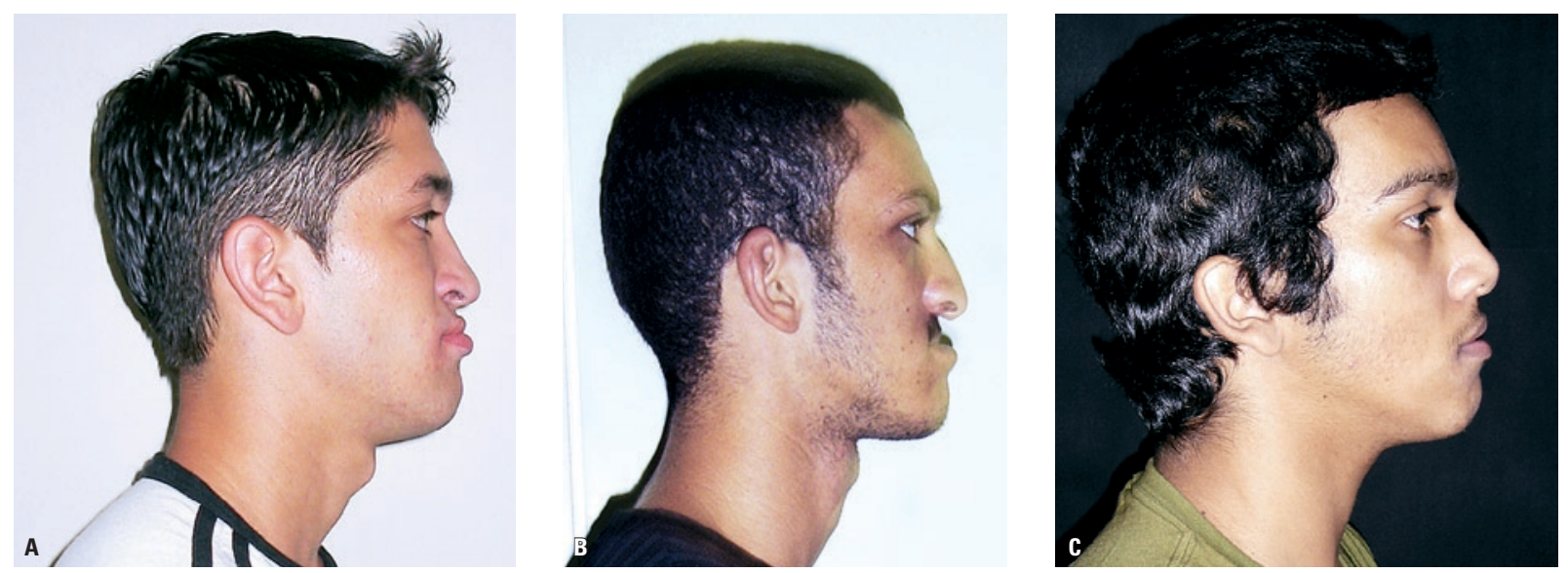

FIGURA 4 - Padrão facial III. A) Deficiência sagital do terço médio. B) Deficiência maxilar associada ao prognatismo mandibular. C) Padrão facial I.
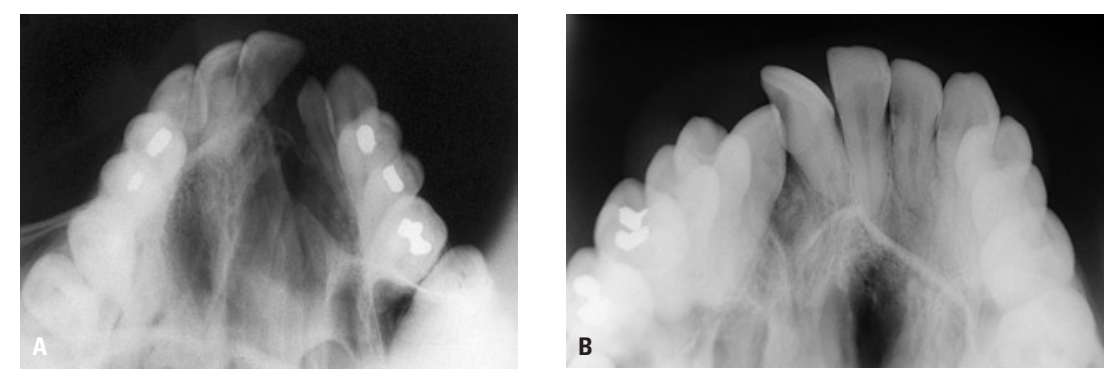

FIGURA 5 - A) Ausência de enxerto ósseo alveolar na área da fissura (Transforame esquerda); B) enxerto ósseo realizado (Transforame direita).

\section{DISCUSSÃO}

A análise dos resultados (Tab. 3) atesta a já conhecida atresia maxilar nos arcos fissurados, quando comparados com a amostra de pacientes normais ${ }^{22}$. A dimensão transversa está diminuída nas quatro áreas de avaliação (3-3, 4-4, 5-5, 6-6) e especialmente concentrada na área de caninos, que apresentou média 31,33\% aquém dos valores considerados normais. Estatisticamente, a fissu- ra provoca maiores alterações na distância entre caninos $^{2}, 10,11,13,14,15,17,18,22,23$ com a área da tuberosidade maxilar funcionando como eixo de rotação na migração medial dos processos palatinos ${ }^{9,10}$. A média da distância entre molares encontrada para o grupo de fissurados corrobora tal afirmação. O valor encontrado foi apenas 11,87\% menor, quando comparado à média do grupo controle.

A segmentação da maxila acaba por fa- 
vorecer a aproximação dos segmentos distintos, independentemente das cirurgias plásticas primárias ${ }^{10,11,14,15,16,29}$. As cirurgias reparadoras primárias, em particular a queiloplastia, parecem ser as maiores responsáveis pela potencialização da atresia maxilar encontrada nos pacientes operados ${ }^{2,7,9,11}$, $12,13,14,17,18,20,23$. Ao final do crescimento, a maxila se mostra atrésica transversalmente e deficiente no sentido ântero-posterior ${ }^{15,17,29}$.

Portadores de fissura não submetidos às cirurgias primárias de queiloplastia e palatoplastia apresentam menor grau de medialização dos processos palatinos ${ }^{9,13,14,18,22}$ (Tab. 4) e, de modo contrário aos pacientes operados, apresentam tendência ao desenvolvimento de má oclusão Classe II de Angle pelo deslocamento anterior da base apical superior ${ }^{1,4,9,13,14,15,18,22}$. No grupo de fissurados desse trabalho, o desenvolvimento de faces Padrão III, foi encontrado em 19 pacientes da amostra (95\% do total) e reflete a restrição de crescimento do complexo nasomaxilar, potencializado pelas cirurgias primárias, atuando fundamentalmente no remodelamento anterior do arco dentário e na diminuição da distância entre caninos. A tendência de crescimento vertical, apresentada pela mandíbula $^{28}$, pode mascarar a deficiência sagital maxilar, e a análise facial, em conjunto com as radiografias de perfil, revelar pacientes Padrão III face longa. A alta prevalência desse tipo facial, juntamente com a atresia maxilar e a mordida cruzada posterior, podem ser consideradas o tripé base para o diagnóstico maxilofacial nos pacientes com fissura transforame incisivo unilateral, e estão em proporção inversa aos achados para grupos de pessoas normais ${ }^{6}$.

De acordo com Mazaheri ${ }^{11}$, já aos 4 anos de idade $90 \%$ dos pacientes com fissura transforame incisivo unilateral apresentam-se com os segmentos palatinos em contato na região alveolar. Adicionalmente, os efeitos da restrição do crescimento se mostram cumulativos ao longo do tempo ${ }^{14,15}$. A ausência do arco maxilar sem contato entre os segmentos palatinos na amostra estudada (Gráf. 1) parece ser justificada pelas afirmações acima, já que, ao nascimento, a dimensão transversa superior se mostra aumentada, quando comparada à de recém nascidos normais ${ }^{1}$. Já que a atresia maxilar se desenvolve de acordo com o crescimento, parece lícito afirmar que essa morfologia específica possa ser encontrada em pacientes infantis, quando a migração medial dos processos palatinos ainda se encontra ativa. A atresia maxilar se relaciona diretamente à mordida cruzada posterior, a má oclusão mais encontrada nos portadores de fissura de lábio e palato ${ }^{3,7,8,9}$. Como nos fissurados a morfologia do arco dentário inferior se encontra preservada, a atresia maxilar é facilmente diagnosticada por meio da relação entre arcos dos modelos de gesso em oclusão.

Tabela 4 - Medidas transversas ( $\mathrm{mm}$ ) para as dimensões entre caninos (3-3), entre primeiros pré-molares (4-4), entre segundos pré-molares (5-5) e entre primeiros molares (6-6), para o grupo TFIU - REVIVA operados de lábio e palato (0), em relação ao grupo TFIU não-operados (NO) ${ }^{23}$ e grupo normal ${ }^{23}$.

\begin{tabular}{|c|c|c|c|c|c|c|}
\hline \multicolumn{7}{|c|}{ dimensão transversa } \\
\hline & \multicolumn{2}{|c|}{ TFIU (0) } & \multicolumn{2}{|c|}{ TFIU (NO) } & \multicolumn{2}{|c|}{ normal } \\
\hline & média & d.p. & média & d.p. & média & d.p. \\
\hline 3-3 & 24,71 & 3,48 & 30,26 & 4,54 & 35,98 & 2,32 \\
\hline $4-4$ & 34,71 & 3,85 & 39,08 & 4,86 & 43,78 & 2,37 \\
\hline $5-5$ & 41,98 & 4,45 & 46,45 & 4,88 & 49,37 & 2,96 \\
\hline $6-6$ & 47,78 & 4,07 & 52,22 & 3,94 & 54,21 & 3,58 \\
\hline
\end{tabular}


Neste estudo, a prevalência de mordida cruzada posterior ao início do tratamento ortodôntico foi de 91,45\%, presente em 19 pacientes da amostra (Gráf. 2), independente do tipo de morfologia maxilar encontrada (Tab. 5). Essa constatação estabelece a mecânica expansionista ortopédica para reposicionamento dos segmentos maxilares como medida primária no processo de reabilitação oclusal2,3,7,8,12,17.

A mordida cruzada total, dado relevante que reflete mais uma vez o pobre potencial de desenvolvimento ântero-posterior e transverso da maxila, foi encontrada em 7 dos 20 pacientes, ou 35\% da amostra avaliada em máxima intercuspidação habitual (MIH). Essa entidade oclusal traz graves repercussões à concavidade facial, pois gera deficiência sagital do terço médio e empobrece a expressão óssea do arco zigomático ${ }^{4,9,12,20}$. De maneira cumulativa à queiloplastia, a palatoplastia total, a partir do primeiro ano de vida, parece influir negativamente no desenvolvimento transverso maxilar, especificamente nas áreas média e posterior, relacionadas aos pré-molares e molares ${ }^{9,18}$. Segundo Silva Filho ${ }^{22}$, seus efeitos são potencializados a partir dos 6 anos de vida, justamente no início do crescimento maxilar, portanto em época oportuna para o início da intervenção ortodôntica.

A mordida cruzada total sugere, ainda, a reabilitação orto-cirúrgica para avanço da maxila nesses pacientes, já que perfis côncavos, do tipo Classe III, padronizam e, mais do que tudo, estigmatizam a face do paciente fissurado. Os dados de Mazotini et al. ${ }^{12}$ mostram que $27 \%$ dos fissurados de palato necessitam de tratamento ortodôntico associado à cirurgia ortognática.
$\mathrm{Na}$ situação ideal, a abordagem ortodôntica inicial deve se relacionar ao desenvolvimento radicular pré-eruptivo do canino permanente adjacente à fissura ${ }^{2,5,7,12,19,27}$. A expansão rápida da maxila (ERM) é geralmente a primeira abordagem e visa a correção da atresia maxilar e mordida cruzada posterior, mas fundamentalmente o estabelecimento da morfologia maxilar apropriada para a cirurgia de enxerto ósseo alveolar secundário. Para tanto, a intervenção deve ser finalizada antes da erupção do canino na cavidade bucal, geralmente entre os 8 e 12 anos de idade ${ }^{2,5,27}$. O enxerto corrige o defeito ósseo alveolar e provê estrutura periodontal de suporte e proteção para o canino permanente, que irromperá na área da fissura, fazendo contato com o incisivo central numa relação oclusal do tipo Classe II $5,24,25$. No entanto, em populações carentes de tratamento especializado, o acompanhamento ao longo do crescimento facial durante a primeira infância e adolescência após as cirurgias primárias é inexistente, impedindo o estabelecimento desse protocolo, ideal ao tratamento reabilitador, e gerando seqüelas faciais com maiores dificuldades de reabilitação.

Essa é a realidade encontrada hoje na população de fissurados em tratamento no REVIVA. Do grupo amostral de pacientes $(\mathrm{n}=20)$, dezoito deles (90\%) se encontravam com o crescimento finalizado ao início do tratamento ortodôntico e, portanto, com as dimensões finais da maxila já estabelecidas, assim como as seqüelas do crescimento. Segundo Pruzansky ${ }^{14,15}$, os efeitos restritivos do crescimento maxilar se acumulam ao longo do tempo, criando grandes seqüelas faciais na vida adulta. Nessas condições, a expansão maxilar é também

Tabela 5 - Relação entre a má oclusão e o tipo de migração medial dos processos palatinos: MCT (Mordida Cruzada Total), MCAPU (Mordida Cruzada Ântero-Posterior Unilateral), MCPU (Mordida Cruzada Posterior Unilateral) e MCPB (Mordida Cruzada Posterior Bilateral).

\begin{tabular}{|c|c|c|c|c|c|c|}
\hline & MCA & Мст & MCAPU & MCPU & МСРВ & n (total) \\
\hline contato & 1 & 4 & 2 & 3 & 2 & 12 \\
\hline colapso & -- & 3 & 3 & 3 & 1 & 10 \\
\hline $\mathrm{n}$ (total) & 1 & 7 & 5 & 6 & 3 & 22 \\
\hline
\end{tabular}


realizada com objetivos semelhantes ao anteriormente citados para a intervenção precoce. Porém, para se alcançar o efeito ortopédico essencial à reabilitação, a mesma deve ser realizada sob assistência cirúrgica, encarecendo e dificultando o tratamento.

O padrão ouro de reabilitação ainda se encontra longe da realidade fluminense. A avaliação das radiografias oclusais mostrou que a reabilitação cirúrgica desses pacientes se limita à manipulação de tecido mole do lábio e palato. Do grupo avaliado, apenas 2 indivíduos (10\%) tiveram realizada a cirurgia de enxerto ósseo alveolar (Gráf. 4). Nessa fase, no entanto, o enxerto é classificado como terciário ou tardio e visa, basicamente, o fechamento de fístulas buconasais e a reabilitação protética do dente, ou dentes, ausentes na área da fissura ${ }^{16,19}$. O prognóstico para essa modalidade de enxerto indica cautela quanto à movimentação dentária em direção à área enxertada. A erupção espontânea do canino é um estímulo à integração óssea no enxerto secundário, e isso determina o diferencial do prognóstico entre enxerto secundário e terciário ${ }^{5,24,25,27}$.

Particularmente importante num ambiente inócuo ao crescimento facial ${ }^{4,14,15,23}$, a alta idade cronológica para o início das atividades ortodônticas (Tab. 2) atua negativamente na ferramenta essencial que o ortodontista tem em mãos para a melhor reabilitação: a atuação em período fértil de crescimento craniofacial.

\section{CONCLUSÃO}

A constatação clínica no departamento de Ortodontia do Centro de Tratamento de Anomalias Craniofaciais do Rio de Janeiro - REVIVA, revela que o tratamento reabilitador vem pecando pelo desacompanhamento dos pacientes a partir do momento em que terminam os procedimentos reabilitadores primários de queiloplastia e palatoplastia ainda nos primeiros anos de vida. Esses mesmos pacientes, ao chegarem já em idade adulta para os procedimentos ortodônticos iniciais, apresentam, como conseqüência da falta de planejamento, severas más oclusões relacionadas ao déficit maxilar, que poderiam ter sido diminuidas e até mesmo solucionadas a partir da segunda infância pelos procedimentos de expansão maxilar e enxerto ósseo alveolar, no final do segundo período transitório da dentadura mista.

A busca por reverter o quadro de seqüelas apresentadas pela população adulta é necessária. Isso é feito por meio do estabelecimento de protocolos tardios coerentes que estabeleçam satisfatoriamente as necessidades estéticas e funcionais dos pacientes.

\title{
Morphological analysis of patients with cleft of lip and palate from Treatment Center of Craniofacial Anomalies of Rio de Janeiro state
}

\begin{abstract}
Aim: To characterize the occlusion and facial pattern to diagnose the rehabilitation requirements of patients with cleft of lip and palate from Center for Treatment of Craniofacial Anomalies of Rio de Janeiro state. Methods: A sample of $20 \mathrm{cleft}$ lip and palate patients, operated of lip and palate, without orthodontic treatment were selected. The transversal dimensions and morphology of maxillary dental arches, as well as facial pattern and inherent characteristics of rehabilitation process were evaluated. Transversal dimensions were compared with a normal occlusion group ( $n=22$ ). Results: Data indicated transverse maxillary deficiency associated to different kinds of cross-bites in the cleft group. Facial profile shows strong tendency for maxillary hypoplasia, while alveolar bone graft was absent in the rehabilitation process. Conclusion: The findings suggest the impact of primary surgeries of lip and palate on the three-dimensional growing of maxilla. The gold pattern of rehabilitation cannot be reached without alveolar bone graft, a therapy that provides unity and integrity to alveolar maxillary bone.
\end{abstract}

Key words: Cleft lip and palate. Dental arch morphology. Dental arch dimensions. 


\section{REFERÊNCIAS}

1. ADUSS, H. Craniofacial growth in complete unilateral cleft lip and palate. Angle Orthod., Appleton, v. 41, p. 202-213, 1971

2. AIELLO, C. A.; SILVA FILHO, O. G.; FREITAS, J. A. S. Fissuras lábio-palatais: uma visão contemporânea do processo reabilitador. In: MUGA-YAR, L. R. F. Pacientes portadores de necessidades especiais: manual de Odontologia e saúde oral. São Paulo: Pancast, 2000.

3. ATHANASIOU, A. E. Frequency of crossbite in surgical treated cleft lip and/or palate children. J. Pedod., Birmingham, v. 10, p. 340-351, 1986.

4. BISHARA, S. E. Facial and dental relationships of individuals with unoperated clefts of the lip and/or palate. Cleft Palate J. Pittsburgh, v. 13, p. 238-252, 1976

5. BOYNE, P. J.; SANDS, N. R. Secondary bone grafting of residual alveolar and palatal clefts. J. Oral Surg., Chicago, v. 30 no. 2, p. 87-92, 1972

6. CAPELOZZA FILHO, L. Diagnóstico em Ortodontia. Maringá: Dental Press, 2004.

7. CAPELOZZA FILHO, L.; ALMEIDA, A. M.; URSI, W. J. S. Rapid maxillary expansion in cleft lip and palate patients. J. Clin. Orthod., Boulder, v. 28, no. 1, p. 34-39, Jan. 1994

8. CAPELOZZA FILHO, L.; MARTINS, R. M.; MAZZOTTINI, R. Análise do diâmetro transverso do maxilar superior de portadores de fissura trans-forame incisivo unilateral. Ars. Curandi Odontol., São Paulo, v. 6, no. 6, p. 42-51, set. 1979.

9. CAPELOZZA FILHO, L.; NORMANDO, A. D. C.; SILVA FILHO, O. G. Isolated influences of lip and palate surgery on facial growth: comparison of operated and unoperated male adults with UCLP. Cleft Palate Craniofac. J., Lewiston, v. 33, no. 1, p. 51-56, Jan. 1996

10. MAZAHERI, M. et al. Changes in arch form and dimensions of cleft patients. Am. J. Orthod., St. Louis, v. 60, p. 22-30, 1967.

11. MAZAHERI, M. et al. Evaluation of maxillary dental arch form in unilateral clefts of lip, alveolus, and palate from one month to four years. Cleft Palate Craniofac. J., Lewiston, v. 30, p. 90-93, 1993

12. MAZZOTTINI, R.; FREITAS, J. A. S.; SILVA FILHO, O. M. A cirurgia ortognática no protocolo de tratamento das fissuras lábio-palatais. In: ARAÚJO, A. Cirurgia ortognática. 4. ed. São Paulo: Ed. Santos, 1999. p. 309-350.

13. NORMANDO, A. D. C.; SILVA FILHO, O. G.; CAPELOZZA FILHO, L. Influence of surgery on maxillary growth in cleft lip and/or palate patients. J. Craniomaxillofac. Surg., Sttutgart, v. 20, no. 3, p. 111-118, Apr. 1992.

14. PRUZANSKY, S. Factors determining arch form in clefts of the lip and palate. Am. J. Orthod., St. Louis, v. 41, p. 827-851, 1955.

15. PRUZANSKY, S. Pre-surgical orthopedics and bone grafting for infants with cleft lip and palate: a dissent. Cleft Palate J. Pittsburgh, v. 1, p. 164-187, 1964

16. SEMB, G. Effect of alveolar bone grafting on maxillary growth in unilateral cleft lip and palate patients. Cleft Palate J., Pittsburgh, v. 25, p. 288-295, 1988

17. SILVA FILHO, O. G.; CAPELOZZA FILHO, L.; WERNECK, V. A. FREITAS, J. A. S. Abordagem ortodôntica ao paciente com fissura unilateral completa de lábio e palato. Ortodontia, São Paulo, v. 31, no. 3, p. 32-44, set./dez. 1998.

18. SILVA FILHO, O. G.; CAPELOZZA FILHO, L.; RAMOS, A. L. Influence of palatoplasty on the upper dental arch shape and dimensions of unilateral complete cleft lip and palate patients. Braz. J. Dysmorp. Speech-Hearing Disorders, Bauru, v. 1, no. 1, p. 41-44, July 1997.

19. SILVA FILHO, O. G.; FERRARI JÚNIOR, F. M.; CAPELOZZA FILHO, L.; ALBUQUERQUE, M. V. P. Enxerto ósseo em pacientes fissurados: realidade e perspectiva. Ortodontia, São Paulo, v. 28, n. 1, p. 34-45, jan./abr. 1995.

20. SILVA FILHO, O. G.; FERRARI JUNIOR, F. M.; CARVALHO, R. M.; MAZZOTTINI, R. A cirurgia ortognática na reabilitação do paciente portador de fissura unilateral completa de lábio e palato. R. Dental Press Ortodon. Ortop. Facial, Maringá, v. 3, n. 4, p. 51-70, jul./ago. 1998
21. SILVA FILHO, O. G.; FERRARI JÚNIOR, F. M.; ROCHA, D. L.; FREITAS, J. A. S. Classificação das fissuras lábio-palatais: breve histórico, considerações clínicas e sugestão de modificação. Rev. Bras. Cir., Rio de Janeiro, v. 82, n. 2, p. 59-65, mar./abr. 1992

22. SILVA FILHO, O. G.; RAMOS, A. L.; ABDO, R. C. C. Morfologia dos arcos dentários em pacientes portadores de fissura de lábio e palato não operados. Ortodontia, São Paulo, v. 24, n. 1, p. 9-17, 1991

23. SILVA FILHO, O. G. RAMOS, A. L:ABDO, R. C. C. The influence of unilateral cleft lip and palate on maxillary dental arch morphology. Angle Orthod., Appleton, v. 62, no. 4, p. 283290, 1992.

24. SILVA FILHO, O. G.; TELES, S. G.; OZAWA, T. O.; CAPELOZZA FILHO, L. Comportamento do canino permanente na área da fissura frente ao enxerto ósseo secundário. R. Dental Press Ortodon. Ortop. Facial, Maringá, v. 4, n. 5, p. 9-19, set./out. 1999.

25. SILVA FILHO, O. G.: TELES, S. G.; OZAWA, T. O.; CAPELOZZA FILHO, L. Secondary bone graft and eruption of the permanent canine in patients with alveolar clefts: literature review and case report. Angle Orthod., Appleton, v. 70, no. 2, p. 174-178, Apr. 2000

26. SPINA, V.; PSILLAKIS, J. M.; LAPA, F. S. et al. Classificação das fissuras lábio-palatinas: sugestão de modificação. Rev. Hosp. Clin. Fac. Méd. S. Paulo, São Paulo, v. 27, p. 5-6, 1972.

27. TRINDADE, I. K.; MAZZOTTINI, R.; SILVA FILHO, O. G.; TRINDADE, I. E. K.; DEBONI, M. C. Z. Long-term radiographic assessment of secondary alveolar bone grafting outcomes in patients with alveolar clefts. Oral Surg. Oral. Med. Oral Pathol. Oral Radiol. Endod., St. Louis, v. 100, no. 3, p. 271 277, Sept. 2005.

28. VALE, D. M. V.: CAPELOZZA FILHO, L.; SILVA FILHO, O. G. O comportamento da mandíbula frente ao crescimento e ao tratamento cirúrgico nas fissuras trans-forame incisivo bilateral. Ortodontia, São Paulo, v. 27, n. 1, p. 4-12, jan./abr. 1994.

29. WADA, T.; MYAZAKI, T. Growth and changes in maxillary arch form in complete unilateral cleft lip and palate children. Cleft Palate J., Pittsburgh, v. 12, p. 115-130, 1975

Endereço para correspondência Alexandre de Almeida Ribeiro

Rua Belisário Távora 221 / 303, Laranjeiras

CEP: 22.245-070 - Rio de Janeiro / RJ

E-mail: ribeiroalexandre@uol.com.br 